\title{
Tagungsbericht
}

\section{arenapublica: Forum und Messe für öffentliche Leistungen}

Eine Plattform für die Begegnung von Staat, Wirtschaft und Gesellschaft sowie Forum und Messe für öffentliche Leistungen möchte seit 2004 alle zwei Jahre die arenapublica sein. Unter wissenschaftlicher Leitung des Direktors des Instituts für Öffentliche Dienstleistungen der Universität St. Gallen (IDT-HSG), Prof. Dr. Kuno Schedler, und in enger organisatorischer Kooperation mit dem Direktor der Olma Messen St. Gallen, Hanspeter Egli, fand die arenapublica erstmals vom 23. bis zum 25. Juni 2004 auf dem Geländer der Olma Messen St. Gallen statt.

Nicht das unberührte Appenzeller Voralpenland, auf welches die Besucher von der Terrasse der Messe- und Ausstellungshalle blicken konnten, sondern die Deutschland, Liechtenstein, Österreich und die Schweiz verbindende Lage in der Euregio Bodensee war der Grund für die Wahl des Veranstaltungsortes. Verdeutlicht wurde dadurch die länderübergreifende, internationale Verankerung der arenapublica als Ort der Begegnung und des Austauschs für den deutschsprachigen öffentlichen Sektor.

Führungskräfte und Angestellte öffentlicher Institutionen, Betriebe und Behörden, Beamte und Mitarbeiter aus Bund, Kantonen/Ländern und Kommunen/Gemeinden, Politiker, Wissenschaftler und Studierende sowie Fachkräfte aus Dienstleistung und Beratung trafen in St. Gallen zusammen, um die vielfältigen Herausforderungen, vor denen öffentliche Institutionen heue stehen, zu identifizieren, kritisch zu diskutieren und mit neuen Ansätzen und Lösungen anzugehen. Die länderübergreifende Verbesserung öffentlicher (Dienst-) Leistungen ist das Ziel. Unterstrichen wurden Bedeutung und Ziel der arenapublica durch ein international zusammengestelltes Patronatskomitee und einen Fachbeirat einerseits, andererseits durch die Einbettung eines ersten internationalen Tages für Bürgermeister aus Baden-Württemberg, Bayern, Vorarlberg, dem Fürstentum Liechtenstein und den Ostschweizer Kantonen sowie der Jahresversammlung der Schweizerischen Gesellschaft für Verwaltungswissenschaften (SGVW), die auch ein eigenes Forum zu wirksamer Kommunikation von Regierung und Verwaltung anbot.

Bereits der mit Interesse erwartete Eröffnungsreferent, der ehemalige schweizerische Botschafter in Berlin Thomas Borer, sprach in seinem Vortrag »Reformen über Reformen: Wie kommt der Staat aus der Krise? « die wunden Punkte in den Verwaltungen an und forderte so die Messeteilnehmer und Referenten heraus. Ohne ein neues Selbstverständnis von Politikern und Parteien könnten keine Reformen angegangen werden, die schließlich Staat und Politik zu strategischem und effizientem Handeln veranlassen würden. Nur Reformmut und die bewusste Übernahme von Führungsverantwortung durch die politisch Verantwortlichen wecke bei den Bürgern Problemsensibilität und Reformbegeisterung.

Prof. Dr. Kuno Schedler unterstrich die Aussagen Borers deutlich, indem er einen »neuen moralischen Straftatbestand der > unterlassenen Führungsleistung < für Führungskräfte in der Verwaltung « forderte. Schedler beschrieb wirkungsorientierte Verwaltungsführung als einen Bausatz von Steuerungs- und Führungselementen, die einer modernen Führungsphilosophie verpflichtet seien. Kernpunkte seien dabei: Führung durch Zielvereinbarung, Delegation und Anreize. Die Modernisierung der Verwaltung baue im Wesentlichen auf den Führungsfähigkeiten und dem Führungswillen von Verantwortungsträgern auf. Forum und Messe seien eine optimale Plattform, um genau diese Fragen zu vertiefen, Schlüsse für die Zukunft abzuleiten und weitere Schritte einzuleiten.

Die arenapublica entstand als eine Kombination aus Messe und Forum. In Ergänzung und als Antwort auf die im Forum aufgegriffenen aktuellen Fragestellungen boten rund achtzig Firmen, Agenturen, (Fach-) Hochschulen, Universitäten und öffentliche Einrichtungen aus Deutschland, Österreich und der Schweiz im Rahmen der Messe Informationen und Einblicke in ihre Tätigkeiten und Produkte an, darunter im öffentlichen Sektor bekannte Namen wie SAP, PricewaterhouseCoopers und die bremen online services $G m b H \& C o$. KG. Das von den Organisatoren selbst festgesetzte Ziel von 70 Ausstellern für diese erste arenapublica wurde übertroffen. Mit dem »Publirama« und mit einer Poster Session wurde den Ausstellern ein eigener Raum für ihre Präsentation geboten.
Angeboten wurden den Besuchern der arenapublica insgesamt fünfzehn Foren in fünf Sessionen. Jede Session wurde von einer für alle Teilnehmer bestimmte dreißigminütige »Key Note« eingeleitet. Hierfür konnten hochrangige Vertreter aus Politik und Wissenschaft gewonnen werden, darunter Bundeskanzlerin Annemarie Huber-Hotz, Prof. Dr. Peter Glotz und Prof. Dr. Ernst Buschor, die in bewusst provokativen Vorträgen Impulse und Denkanstöße für die nachfolgenden Foren lieferten.

Die Foren befassten sich mit folgenden Themen:

- Die Organisation von Informationstechnologie in öffentlichen Verwaltungen

- Demenz/Alter als Herausforderung für Pflegeorganisationen

- Gebietsreformen im deutschen Sprachraum: Wie weit kann man gehen?

- Open Source-Technologien für öffentliche Verwaltungen

- Qualitätsmanagement in Gesundheitsorganisationen

- Wirksame Kommunikation von Regierung und Verwaltung (SGVWForum)

- Städtische Sicherheit - Zusammenarbeit tut Not

- Finanzielle Führung und Controlling in Gesundheitsorganisationen

- Ehrenamt hat Zukunft: Wie die Aktivierung der Bürger wirklich funktionieren kann

- Gebäudemanagement/Real Estate-Management für die öffentlichen Institutionen

- Aktuelle Entwicklungen im öffentlichen Rechnungswesen und Verwaltungscontrolling

- (Zer)Stört Electronic Government den Föderalismus?

- Probleme eines (mangelnden) Gesamtkonzepts der Führung im Schulwesen

- Die Auswirkungen des Wettbewerbs auf die Non-Profit-Organisationen

- Neue Rollen des Staates: Eigentümer, Kontraktpartner, Gewährleister.

Jedes Forum begann mit drei bis vier zwanzig- bis dreißigminütigen Referaten. Ausführliche Diskussion der dargestellten Ansichten, Probleme und Lösungsansätze schlossen sich an, die bisweilen kontrovers und leidenschaftlich geführt wurden. Bei einigen Diskussionen machte sich allerdings auch der geringe Teilnehmerzuspruch bemerkbar, der mit rund fünfhundert Forumsteilnehmern und cirka 1.400 Messebesuchern doch weit unter den Erwartungen der Organisatoren lag. Trotz der wissenschaftlich hochkarätigen Zusammensetzung und des großen Engagements der Referenten in den Foren konnten manche Tagungsräume nur teilweise gefüllt werden. Die überwiegend österreichischen und schweizerischen Teilnehmer lassen dabei vermuten, dass Dienstreisen in die Schweiz derzeit in Deutschland nur schwer zu begründen sind. Gleichwohl waren viele Referenten aus Deutschland*

Wichtigstes Ergebnis der arenapublica 2004 war für sicher alle Teilnehmer die Erkenntnis, dass die Probleme in den Bodensee-Anrainerstaaten trotz aller verwaltungshistorischen, verwaltungsrechtlichen und verwaltungskulturellen Unterschiede zumindest im Kern vergleichbar sind. Eine intensivere länderübergreifende Zusammenarbeit zur Bewältigung der aktuellen Herausforderungen im Sinne eines New Public Management könnte, über die gegenseitige fachliche Befruchtung hinaus, auch Effizienzgewinne erbringen, eventuell sogar die dringend erhofften Kosteneinsparungen. Insofern ist auch die arenapublica, neben anderen wie der Berliner Messe »Moderner Staat«, ein Gewinn im Messegeschehen.

Abschließend ist noch das große und perfekte Engagement der Organisatoren hervorzuheben, das - nach eingehender Analyse der diesjährigen Veranstaltung - sicher zu einer erfolgreichen Wiederauflage der arenapublica 2006 in St. Gallen führt.

Markus Städler, Mag. rer. publ., Regierungsrat z.A. beim Bundesverwaltungsamt in Köln

* Ein Gesamtprogramm mit allen Referenten ist im Internet abrufbar unter: http://www.arenapublica.org 